\title{
3D Curves Reconstruction from Multiple Images
}

\author{
F. Mai and Y.S. Hung \\ Dept. of Electrical and Electronic Engineering \\ The University of Hong Kong \\ Hong Kong \\ feimai@eee.hku.hk,yshung@eee.hku.hk
}

\begin{abstract}
In this paper, we propose a new approach for reconstructing 3D curves from a sequence of $2 D$ images taken by uncalibrated cameras. A curve in 3D space is represented by a sequence of $3 \mathrm{D}$ points sampled along the curve, and the 3D points are reconstructed by minimizing the distances from their projections to the measured 2D curves on different images (i.e., 2D curve reprojection error). The minimization problem is solved by an iterative algorithm which is guaranteed to converge to a (local) minimum of the $2 \mathrm{D}$ reprojection error. Without requiring calibrated cameras or additional point features, our method can reconstruct multiple 3D curves simultaneously from multiple images and it readily handles images with missing and/or partially occluded curves.
\end{abstract}

Keywords-3D curve reconstruction; partial occlusion; uncalibrated cameras

\section{INTRODUCTION}

The recovery of a 3D scene from a sequence of $2 \mathrm{D}$ images is a central problem in computer vision. A large body of existing research has been devoted to $3 \mathrm{D}$ reconstruction of low-level features such as point features, where a 3D scene is represented by a point cloud [1][2]. However, a wireframe model is a more effective way of presenting a 3D scene than a point cloud. A wireframe model is mainly made up of lines, circles (or ellipse) and curves. There have been some efforts on reconstruction of lines and ellipses from multiple views [3][4][5][6], but very little work has been done on general curve reconstruction.

This is because it is much harder to reconstruct general curves than other features like points, line segments and ellipses, due to the following difficulties: 1) the analytical form of a general curve is not directly available; 2) the point correspondences along curves are not known such that standard point reconstruction methods are not applicable; 3 ) missing (or partially occluded) curves in images are possible due to failure of feature extraction or occlusion.

Attempts have been made by Kaminski et al. in reconstructing general algebraic curves both for planar [7] and non-planar cases [8]. They developed reconstruction algorithms by extending Kruppa's equation to general curves, assuming known camera matrices. Berthilsson et al. extended the concept of affine shape in [9], and applied the notion to develop an algorithm for reconstructing general 3D curves [10]. The algorithm relies on aligning the parameterizations of matched curves so that the points traced on each curve become corresponding points. Although this algorithm works with any number of images taken by uncalibrated cameras, it is not directly applicable for reconstruction of multiple curves from multiple views with missing and/or partially occluded curves. To avoid the difficulties in the curve reconstruction problem, $\mathrm{Wu}$ and $\mathrm{Yu}$ [11] and Zheng et al. [12] adopted fully interactive schemes to recover 3D scene from calibrated images. In the semi-automatic curve reconstruction process, corner point (or marker) correspondences and curve correspondences are required to be input by users. Camera poses and epipolar geometry are estimated using standard techniques in computer vision [13]. Then point correspondences along curves are obtained by imposing the epipolar constraint. Finally the 3D points associated with the $2 \mathrm{D}$ point correspondences along curves are reconstructed using bundle adjustment, and 3D curves are represented by the lists of reconstructed 3D points.

In general, existing curve reconstruction methods suffer from one or more of the following drawbacks:

(1) requiring calibrated cameras;

(2) a lack of provision for missing and/or partially occluded curves;

(3) relying on additional information like corner point correspondences.

In this paper, we propose a point-based curve reconstruction method that addresses all of the above problems. In our approach, a 2D (or 3D) curve is represented as a sequence of $2 \mathrm{D}$ (or 3D) points, and the $3 \mathrm{D}$ curves are reconstructed by minimizing the $2 D$ curve reprojection error, which is defined as the geometric distance from the reprojected 3D points (along the 3D curves) to the measured 2D curves. Given curve correspondences in images, our method does not require any other additional information of the image features or the cameras, and the problem of missing and/or partially occluded curves is readily handled.

The paper is organized as follows. In Section II, the curve reconstruction problem is reformulated as a minimization problem, and the proposed algorithm for solving this minimization problem is presented in Section III. Experimental results are given in Section IV to demonstrate the performance of the proposed method. Concluding remarks are given in Section V. 


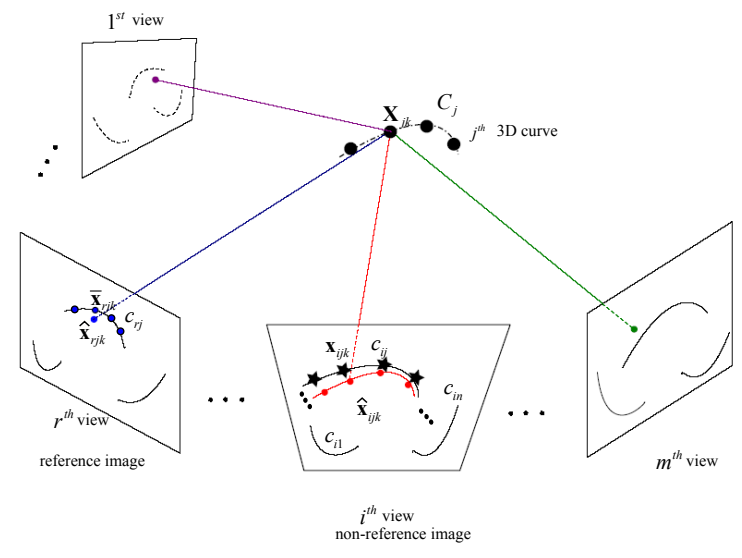

Figure 1. The 3D curve reconstruction from $m$ images

\section{PROBLEM FORMULATION}

The basic idea of the proposed curve reconstruction algorithm is to minimize the $2 \mathrm{D}$ reprojection error of the curves. Therefore, a critical issue of the proposed curve reconstruction method is how to measure the $2 \mathrm{D}$ reprojection error of curves: the error between a measured curve and its reprojected curve. In the proposed algorithm, a 3D curve is represented by a sequence of 3D points, called representative points, sampled along the $3 \mathrm{D}$ curve. We use the distance from the reprojected representative points to its corresponding measured 2D curve as the error measurement, which is explained as follows.

As shown in Figure 1, suppose we are given $m$ views of $n$ 3D curves. The $j^{\text {th }}(j=1,2, \cdots, n) 3 \mathrm{D}$ curve $C_{j}$ is to be reconstructed using $N_{j}$ 3D representative points along the curve, denoted as $X_{j k}=\left[U_{j k} V_{j k} W_{j k} 1\right]^{T}$ in homogeneous coordinate $\left(k=1,2, \cdots, N_{j}\right)$, and shown as black dots in Figure 1.

Let the 2D images of the $j^{t h} 3 \mathrm{D}$ curve on the $i^{t h}$ $(i=1,2, \cdots, m)$ view be measured as $c_{i j}$. Let $N_{j}$ points $x_{i j k}=\left[\begin{array}{lll}u_{i j k} & v_{i j k} & 1\end{array}\right]^{T}$ (black stars in Figure 1) on $c_{i j}$ be its $2 \mathrm{D}$ representative points, which are the corresponding images of the 3D points $X_{j k}$. Suppose the 3D point $X_{j k}$ is projected onto the $i^{\text {th }}$ view with projection matrices $P_{i}(i=1,2, \cdots, m)$ as $\hat{x}_{i j k}$ (red dots in Figure 1), i.e.,

$$
\lambda_{i j k} \hat{x}_{i j k}=P_{i} X_{j k}
$$

where $\lambda_{i j k}$ represents the depth of $X_{j k}$ measured along the optical axis of the $i^{t h}$ camera. Ideally, $X_{j k}$ should project to a point lying on $c_{i j}$ in the $i^{t h}$ view, in which case $x_{i j k}$ can be taken to be $\hat{x}_{i j k}$. In practice, $\hat{x}_{i j k}$ will not lie exactly on $c_{i j}$ due to noise or other measurement errors. In this case, $x_{i j k}$ will be taken to be the point lying on $c_{i j}$ closest to $\hat{x}_{i j k}$. Hence, the idea of the proposed method to reconstructing $C_{j}$ is to reconstruct all its representative points $X_{j k}$, together with the projection matrix $P_{i}$ so that their projections $\hat{x}_{i j k}$ on all the views are as close as possible in a geometric sense to the corresponding measured curve $c_{i j}$ in the 2D images.

Since it is possible that some curves are missing or partially occluded in images due to failure of feature extraction or occlusion, we define the the $2 \mathrm{D}$ curve reprojection error with the observed curves (or partial curves) only. Let us define an index set for the representative points on the measured 2D curves (or partial curves) as:

$$
\begin{aligned}
\mathbf{A}= & \left\{(i, j, k) \mid \text { the } k^{t h}\right. \text { representative point on } \\
& \text { the } \left.j^{\text {th }} \text { curve is observed in the } i^{t h} \text { view }\right\}
\end{aligned}
$$

Then the 2D reprojection error measured for the curves can be defined as the summation of squared distances from the reprojected point $\hat{x}_{i j k}$ to the measured 2D curve $c_{i j}$ (denoted as $d_{i j k}$ ), over all the observed 2D representative points:

$$
\begin{gathered}
d=\sum_{(i, j, k) \in \mathbf{A}} d_{i j k}^{2}=\sum_{\begin{array}{c}
(i, j, k) \in \mathbf{A} \\
\text { subject to } x_{i j k} \in c_{i j}
\end{array}} \min _{x_{i j k}}\left\|x_{i j k}-\hat{x}_{i j k}\right\|^{2} \\
\text { substis }
\end{gathered}
$$

Then the simultaneous reconstruction of the $3 \mathrm{D}$ curves and the projection matrices can be formulated by means of a minimization of the 2D curve reprojection error $d$ defined in (3).

\section{Curve Reconstruction}

In this section, we will show how the $2 \mathrm{D}$ curve reprojection error $d$ defined in (3) can be minimized by means of an iterative procedure.

\section{A. Setting reference curves}

Our objective is to reconstruct the 3D curves by minimizing the $2 \mathrm{D}$ reprojection error defined in (3). However, a minimization problem formulated directly from (3) without extra constraints on representative points will not provide a reasonable solution. This is because it is always easier to fit a single point than a sequence of representative points on a curve to the projection model, and therefore the representative points on a 3D curve will tend to converge (if unconstrained) to one single point whose projections are closest to the measured curves.

To avoid the above issue from happening, we make use of the idea of a reference curve. For each curve $C_{j}$ in 3D space, we take its projection on one of the images, say $c_{r j}$, on the $r^{t h}$ view as the reference curve (see Figure 1). Then, we select $N_{j}$ fixed representative points on it $\bar{x}_{r j k}=\left[\bar{u}_{r j k} \bar{v}_{r j k} 1\right]^{T},\left(k=1,2, \cdots, N_{j}\right)$, called reference representative points (blue dots on $c_{r j}$ in Figure 1), which are reasonably separated from each other along the curve. The reconstructed 3D points $X_{j k}$ corresponding to $\bar{x}_{r j k}$ will be taken as 3D representative points of $C_{j}$. Let us define the 
index set for the representative points on the reference curve as

$$
\begin{aligned}
\mathbf{R}= & \left\{(r, j, k) \mid \bar{x}_{r j k}\right. \text { are sampled from the reference } \\
& \text { curve } \left.c_{r j} \text { as representative points }\right\}
\end{aligned}
$$

In the minimization process, we require the $N_{j}$ reprojected representative points $\hat{x}_{r j k}$ of the $3 \mathrm{D}$ curve $C_{j}$ on the reference curve to approach the fixed reference representative points $\bar{x}_{r j k}$, while the representative points $x_{i j k}$ on any non-reference curves are free to move along their corresponding curve $c_{i j}(i \neq r)$. The fixing of the reference representative points can prevent the representative points from converging into one single point.

\section{B. Cost function for the curve reconstruction}

To define the cost function for the curve reconstruction that takes into account all the above considerations, we will rewrite the expression (3) in the form of a cost function suitable for minimization. We will use the following notations.

- $\mathbf{P}=\left[\begin{array}{llll}P_{1}^{T} & P_{2}^{T} & \cdots & P_{m}^{T}\end{array}\right] \in \mathcal{R}^{3 m \times 4}$ is the joint projection matrix.

- $\mathbf{X}=\left\{X_{j k}\right\},\left(j=1,2, \cdots, n ; k=1,2, \cdots, N_{j}\right)$ is the set containing all the reconstructed $3 \mathrm{D}$ representative points.

- $\beta=\left\{\beta_{i j k}\right\},((i, j, k) \in \mathbf{A}$ is the set of inverse depth of all $3 \mathrm{D}$ representative points from all the views where they are visible.

- $\mathbf{x}=\left\{x_{i j k}\right\},((i, j, k) \in \mathbf{A} \backslash \mathbf{R})$ is the set containing all the representative $2 \mathrm{D}$ images on the non-reference curves.

Let $P_{i}^{l}$ denote the $l^{\text {th }}$ row of $P_{i}(l=1,2,3)$. The 3D curve reconstruction problem minimizing the cost function defined in (3) can now be reformulated as (5):

$$
\begin{gathered}
\min _{\mathbf{P}, \mathbf{X}, \lambda, \mathbf{x}}\left\{\sum_{(r, j, k) \in \mathbf{R}}\left[\left(\bar{u}_{r j k}-\frac{P_{r}^{1} X_{j k}}{\lambda_{r j k}}\right)^{2}+\left(\bar{v}_{r j k}-\frac{P_{r}^{2} X_{j k}}{\lambda_{r j k}}\right)^{2}\right]\right. \\
\left.\sum_{(i, j, k) \in \mathbf{A} \backslash \mathbf{R}}\left[\left(u_{i j k}-\frac{P_{i}^{1} X_{j k}}{\lambda_{i j k}}\right)^{2}+\left(v_{i j k}-\frac{P_{i}^{2} X_{j k}}{\lambda_{i j k}}\right)^{2}\right]\right\}
\end{gathered}
$$

subject to

$$
\begin{aligned}
& \text { (a) } x_{i j k}=\left[\begin{array}{lll}
u_{i j k} & v_{i j k} & 1
\end{array}\right]^{T} \in c_{i j},(i, j, k) \in \mathbf{A} \backslash \mathbf{R} \\
& \text { (b) } \lambda_{i j k}=P_{i}^{3} X_{j k},(i, j, k) \in \mathbf{A}
\end{aligned}
$$

where the first term $\left[\left(\bar{u}_{r j k}-\frac{P_{r}^{1} X_{j k}}{P_{r}^{3} X_{j k}}\right)^{2}+\left(\bar{v}_{r j k}-\frac{P_{r}^{2} X_{j k}}{P_{r}^{3} X_{j k}}\right)^{2}\right]$ corresponds to the 2D reprojection error for all the reference curves, and the second term $\left[\left(u_{i j k}-\frac{P_{i}^{1} X_{j k}}{\lambda_{i j k}}\right)^{2}+\left(v_{i j k}-\frac{P_{i}^{2} X_{j k}}{\lambda_{i j k}}\right)^{2}\right] \quad$ corresponds to the 2D reprojection error for all the non-reference curves. During the minimization process, $x_{i j k}$ will move along $c_{i j}$ following the movement of $\hat{x}_{i j k}$ due to the adjustment of $P_{i}$ and $X_{j k}$, while $\bar{x}_{r j k}$ will remain fixed on $x_{r j k}$. Therefore, by fixing $\bar{x}_{r j k}$ on curve $c_{r j}$, the first term measures the point-to-point distances between the reprojected point $\hat{x}_{r j k}$ and the measured representative point $\bar{x}_{r j k}$; by allowing $x_{i j k}$ moving along curve $c_{i j}$, the second term measures the point-to-curve distances between the reprojected point $\hat{x}_{i j k}$ and the measured 2D curve $c_{i j}$.

To avoid the nonlinearity in the cost function, the above minimization problem can be reformulated as (6) by introducing the inverse depth $\beta_{i j k}=1 / \lambda_{i j k}$ [14] [6].

$$
\begin{aligned}
\min _{\mathbf{P}, \mathbf{X}, \beta, \mathbf{x}} & \left\{\sum _ { ( r , j , k ) \in \mathbf { R } } \left[\left(\bar{u}_{r j k}-\beta_{r j k} P_{r}^{1} X_{j k}\right)^{2}\right.\right. \\
+ & \left.\left(\bar{v}_{r j k}-\beta_{r j k} P_{r}^{2} X_{j k}\right)^{2}\right] \\
& \sum_{(i, j, k) \in \mathbf{A} \backslash \mathbf{R}}\left[\left(u_{i j k}-\beta_{i j k} P_{i}^{1} X_{j k}\right)^{2}\right. \\
+ & \left.\left.\left(v_{i j k}-\beta_{i j k} P_{i}^{2} X_{j k}\right)^{2}\right]\right\}
\end{aligned}
$$

subject to

$$
\begin{aligned}
& \text { (a) } x_{i j k}=\left[\begin{array}{lll}
u_{i j k} & v_{i j k} & 1
\end{array}\right]^{T} \in c_{i j},(i, j, k) \in \mathbf{A} \backslash \mathbf{R} \\
& \text { (b) } \beta_{i j k} P_{i}^{3} X_{j k}=1,(i, j, k) \in \mathbf{A}
\end{aligned}
$$

By imposing a penalty $\gamma$, we incorporate the constraints (6 (b)) into the cost function given in (6). By letting the penalty $\gamma$ approach $\infty$ in a controlled manner, the constrained minimization problem (6) can be transformed into a sequence of minimization sub-problems defined as (7). The solutions of the minimization sub-problems are proved to converge to the original minimization problem (6) when the penalty approaches infinity [14] [6].

$$
\begin{aligned}
& \min _{\mathbf{P}, \mathbf{X}, \beta, \mathbf{x}} F(\mathbf{P}, \mathbf{X}, \beta, \mathbf{x} ; \gamma) \\
& =\sum_{(r, j, k) \in \mathbf{R}}\left\|\bar{\gamma}_{r j k} \circ\left(\bar{x}_{r j k}-\beta_{r j k} P_{r} X_{j k}\right)\right\|^{2} \\
& +\sum_{(i, j, k) \in \mathbf{A} \backslash \mathbf{R}}\left\|\bar{\gamma}_{i j k} \circ\left(x_{i j k}-\beta_{i j k} P_{i} X_{j k}\right)\right\|^{2} \\
& \text { subject to } \\
& x_{i j k} \in c_{i j},(i, j, k) \in \mathbf{A} \backslash \mathbf{R}
\end{aligned}
$$

where $\circ$ denotes the Hadamard product (given matrices $A=\left[a_{i j}\right]$ and $B=\left[b_{i j}\right]$ of the same size, $A \circ B=\left[a_{i j} b_{i j}\right]$ ). $\bar{\gamma}_{i j k}=\left[\begin{array}{lll}1 & 1 \gamma \eta_{i j k}\end{array}\right]^{T}$, and the weighting factor $\eta_{i j k}$ is used to balance the magnitude of the pixel coordinates with the last element (i.e., unit magnitude) of the homogeneous coordinates. We choose $\eta_{i j k}$ as suggested in [14] and [6]:

$$
\eta_{i j k}=\max \left(\left|u_{i j k}\right|,\left|v_{i j k}\right|\right), \forall(i, j, k) \in \mathbf{A}
$$

\section{Point-based curve reconstruction algorithm}

Given a sequence of $m$ images with $n$ curve correspondences $c_{i j}$ detected in images, we first select the reference curves $c_{r j}$, and sample representative points $\bar{x}_{r j k}$ on $c_{r j}$. 
An iterative procedure is developed to estimate the unknown parameters: $\mathbf{P}, \mathbf{X}, \beta, \mathbf{x}$.

In the proposed iterative procedure, the minimization problem (6) is solved as a sequence of minimization subproblems (7) corresponding to increasing values of $\gamma$. For any fixed $\gamma$, we can solve for one of the four variables $\mathbf{P}, \mathbf{X}, \beta$ and $\mathbf{x}$ as a free parameter while fixing the other three variables, alternately and iteratively until convergence. Note that the constraints in (7) are only related to the variable $\mathbf{x}$ and independent of the other three variables $\mathbf{P}, \mathbf{X}, \beta$. Therefore, $F(\mathbf{P}, \mathbf{X}, \beta, \mathbf{x} ; \gamma)$ is trilinear in $\mathbf{P}, \mathbf{X}, \beta$, and the minimization sub-problem (7) with respect to each of the three variables is a weighted linear least-squares problem solvable by standard techniques. As for the variable $\mathbf{x}$, when all the other three variables are fixed, the minimization with respect to $\mathrm{x}$ amounts to finding a point lying on a given (non-reference) curve $x_{i j k} \in c_{i j},(i, j, k) \in \mathbf{A} \backslash \mathbf{R}$, which is as close as possible to a given point $\hat{x}_{i j k}$.

The following Algorithm 1 gives details about the iterative procedure for solving (6).

The above algorithm is guaranteed to converge to a (local) minimum of the cost function with a geometric meaning given in (6), and a detailed proof is given in [14] [6]. Furthermore, the minimization problem is formulated on the observed data only, and therefore the proposed algorithm is capable of handling the missing and/or occluded curve problem.

\section{Initialization}

In the proposed algorithm, which solves the minimization problem iteratively, a good initial guess is important. Here we apply the method for affine-invariant shape matching [15] to get the initial point correspondences $\mathbf{x}^{(0)}$ on the nonreference curves. An overview of the affine-invariant shape matching method is given as follows, and the details can be found in [15].

Since a perspective transformation between two images of an object can be approximated by an affine transformation if the object is planar and far away from the image plane. The proposed method in [15] measures the similarity (up to an affine transformation), and estimates the affine transformation between a pair of corresponding curves. Given a pair of curves, the matching process consists of four steps as follows.

(1) Affine-invariant points (along each curve) detection. The affine-invariant points, which are preserved under affine transformations, are detected based on the properties of curvature scale space (CSS) shape descriptor [16]. Every two consecutive affine-invariant points determine an affine-invariant segment along the curve, and thus a sequence of ordered affine-invariant segments are created for each curve.

(2) Affine-invariant segments matching. The two sequences of affine-invariant segments are aligned by \begin{tabular}{l} 
Algorithm 1 3D curve reconstruction \\
\hline GIVEN $m$ images with $n$ curve correspondences $c_{i j}$
\end{tabular}

(I) Select the reference curves $c_{r j}$ and set $2 \mathrm{D}$ representative points $\bar{x}_{r j k} \in c_{r j},(r, j, k) \in \mathbf{R}$ on it.

(II) Initialization for $\mathbf{P}^{(0)}, \mathbf{X}^{(0)}, \beta^{(0)}$ (A method for initialization is given in Section III-D).

(III) Iterative minimization process.

(a) Set intial parameters: $\gamma^{(0)}=1, s=0, r=$ $0, e^{(0)}=\epsilon^{(0)}=\infty$.

(b) Put $s=s+1$.

(c) Fix $\mathbf{P}^{(s-1)}, \mathbf{X}^{(s-1)}, \beta^{(s-1)}$, and determine $\mathbf{x}^{(s)}$ by solving

$$
\min _{\mathbf{x}^{(s)}} F\left(\mathbf{P}^{(s-1)}, \mathbf{X}^{(s-1)}, \beta^{(s-1)}, \mathbf{x}^{(s)} ; \gamma^{(r)}\right)
$$

subject to $x_{i j k} \in c_{i j}$.

(d) Fix $\mathbf{P}^{(s-1)}, \mathbf{X}^{(s-1)}, \mathbf{x}^{(s)}$, and determine $\beta^{(s)}$ by solving

$$
\min _{\beta^{(s)}} F\left(\mathbf{P}^{(s-1)}, \mathbf{X}^{(s-1)}, \beta^{(s)}, \mathbf{x}^{(s)} ; \gamma^{(r)}\right) .
$$

(e) Fix $\mathbf{P}^{(s-1)}, \beta^{(s)}, \mathbf{x}^{(s)}$, and determine $\mathbf{X}^{(s)}$ by solving

$$
\min _{\mathbf{X}^{(s)}} F\left(\mathbf{P}^{(s-1)}, \mathbf{X}^{(s)}, \beta^{(s)}, \mathbf{x}^{(s)} ; \gamma^{(r)}\right) .
$$

(f) Fix $\mathbf{X}^{(s)}, \beta^{(s)}, \mathbf{x}^{(s)}$, and determine $\mathbf{P}^{(s)}$ by solving

$$
\epsilon^{(s)}=\min _{\mathbf{P}^{(s)}} F\left(\mathbf{P}^{(s)}, \mathbf{X}^{(s)}, \beta^{(s)}, \mathbf{x}^{(s)} ; \gamma^{(r)}\right) .
$$

(g) Repeat (b)-(f) until $\epsilon^{(s)}$ converges.

(h) Put $r=r+1, e^{(r)}=\epsilon^{(s)}$, $\left(\mathbf{P}^{(0)}, \mathbf{X}^{(0)}, \beta^{(0)}, \mathbf{x}^{(0)}\right)$ $\left(\mathbf{P}^{(s)}, \mathbf{X}^{(s)}, \beta^{(s)}, \mathbf{X}^{(s)}\right)$, $\gamma^{(r)}=1.1 \gamma^{(r-1)}$. Set $s=0$.

(i) Repeat (b)-(h) until $e^{(r)}$ converges.

(IV) Output the solution $\left(\mathbf{P}^{*}, \mathbf{X}^{*}, \beta^{*}, \mathbf{x}^{*}\right)=$ $\left(\mathbf{P}^{(s)}, \mathbf{X}^{(s)}, \beta^{(s)}, \mathbf{x}^{(s)}\right)$.

the Smith-Waterman algorithm [17], and thus an initial matched part is obtained.

(3) Matching section extension. If at least one curve is partially visible, the initial matched section is extended using the affine invariant affine-length, such that the maximum matched section is obtained. Accordingly, the point correspondences are obtained and the affine transformation is estimated.

(4) Similarity score computation. Transform the first curve by the estimated affine transformation, and the similarity score between the second curve and the transformed first curve is computed by the standard crosscorrelation technique. 
The above matching method makes use of the global information of the curves, as well as the local information. Therefore, it is capable of matching curves under partial occlusion. The point correspondences obtained from the matching method will provide us a reasonable guess of $\mathbf{x}^{(0)}$. Then the projective point reconstruction algorithm [6], which relies on the initial point correspondences only, is applied to get the initial projection matrices $\mathbf{P}^{(0)}$, 3D representative points $\mathbf{X}^{(0)}$, and inverse depth $\beta^{(0)}$.

\section{EXPERIMENTAL RESULTS}

We have tested our proposed algorithm for curve reconstruction on real images. One example is given in this section. Three images (shown in Figure 2) of a 3D scene are taken by a Canon EOS D30 digital camera, with different focal lengths. Two curves in each image are extracted, each in the form of a sequence of connected edge points, using a level set based method [18]. In this image sequence, the curves on the first and third images are partially occluded. For every two adjacent images, we apply the affine-invariant curve matching method [15] on the curves in a pair-wise manner to create a matching table containing the similarity scores of all the curve pairs. Then curve correspondences are obtained by applying the winner-takes-all technique to the matching table. After that, we select the curves on the second image as the reference curves, and sample the representative points along the reference curves with an interval of 10 points (see Figure 2), resulting in 127 and 173 representative points on the two curves. Finally we run Algorithm 1 on the measured curves correspondences. Figure 3 gives the reconstructed 3D curves (and the position of the cameras), which is a reasonable representation of the real 3D curves. Due to space limitation, we only show the reprojected representative poins on the third image in Figure 4 for visual evaluation. The reprojected representative points are almost lying on the measured curves, which means the $2 \mathrm{D}$ curve reprojection error is small. For numerical evaluation, the mean 2D curve reprojection error (averaged over all reprojected representative points) is 1.78 pixel (with respect to the image size of $1440 \times 2160$ ).

\section{CONCLUSIONS}

In this paper, we propose a new approach for reconstructing 3D curves from a sequence of images taken by uncalibrated cameras. We reconstruct a 3D curve by minimizing the distance of reprojected representative points from the measured 2D curves on different images. By solving a sequence of minimization sub-problems, the proposed iterative algorithm is guaranteed to converge to a (local) minimum of the $2 \mathrm{D}$ curve reprojection error. The proposed method does not require prior calibration of the cameras or additional information of feature points, and it readily handles cases where not all curves are visible or some curves are partially occluded.
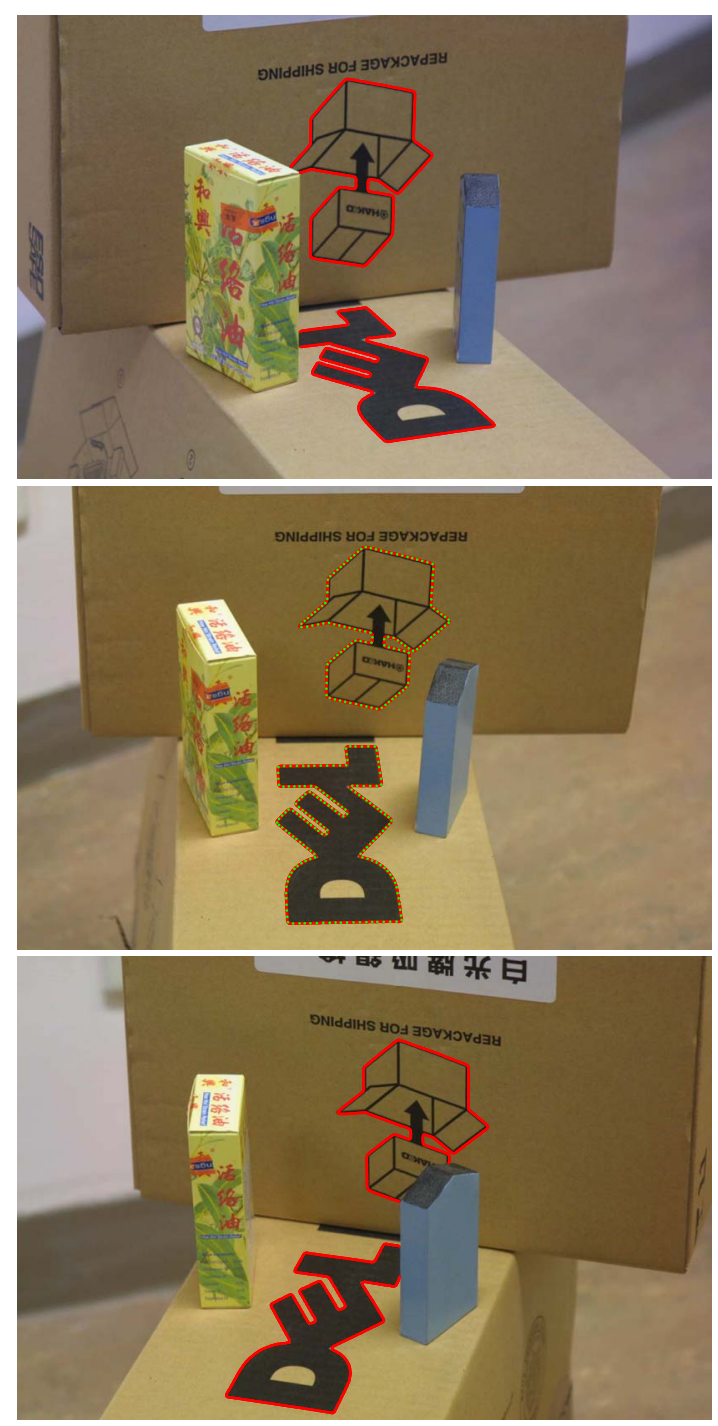

Figure 2. The sequence of images. The extracted curves are shown in red. The curves in the second image are selected as reference curves and the green dots are the representative points.

\section{ACKNOWLEDGMENT}

The work described in this paper was supported by a grant from the Research Grants Council of the Hong Kong Special Administrative Region, China (Project No. HKU 712808E) and CRCG of the University of Hong Kong.

\section{REFERENCES}

[1] P. Sturm and B. Triggs, "A factorization based algorithm for multi-image projective structure and motion," in $E C C V$. Springer-Verlag, 1996, pp. 709-720.

[2] C. Tomasi, "Shape and motion from image streams under orthography: a factorization method," International Journal of Computer Vision, vol. 9, pp. 137-154, 1992. 

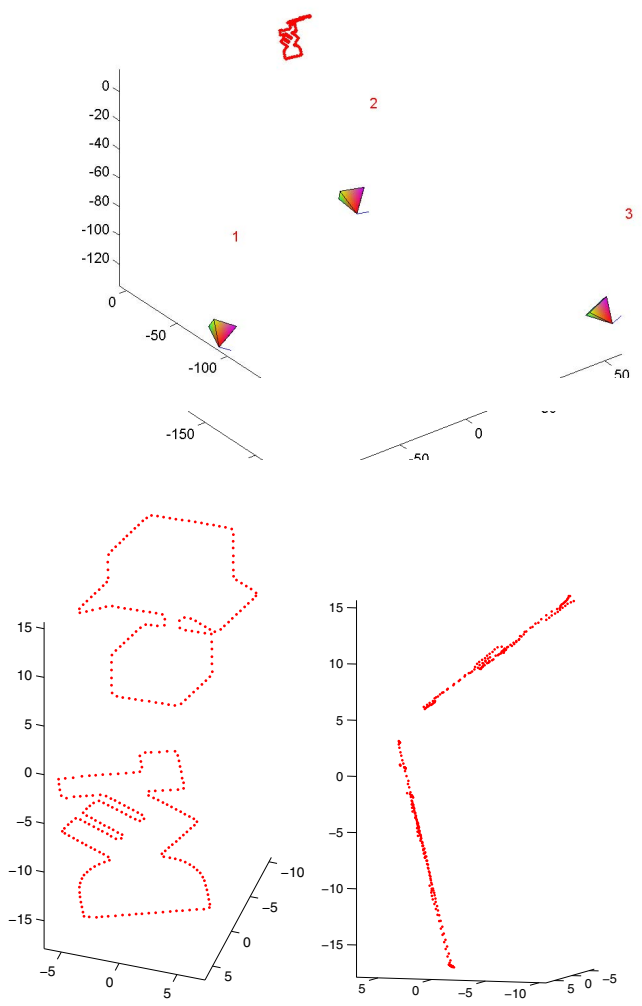

Figure 3. Reconstructed 3D curves. The upper one shows the reconstructed 3D curve and camera positions, and the lower one shows two close views of the reconstructed 3D curves.

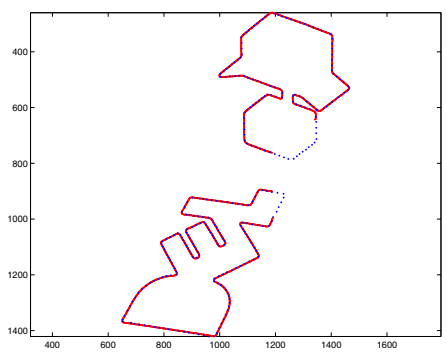

Figure 4. Reprojected representative points on the third image. The red curves are the measured curves, and the blue dots are the reprojected representative points.

[3] R. I. Hartley, "Projective reconstruction from line correspondences," in In Proc. IEEE Conf. on Computer Vision and Pattern Recognition. IEEE Computer Society Press, 1994, pp. 903-907.

[4] - "Lines and points in three views and the trifocal tensor," International Journal of Computer Vision, vol. 22, no. 2, pp. 125-140, 1997.

[5] L. Quan, "Conic reconstruction and correspondence from two views," IEEE Trans. Pattern Anal. Mach. Intell., vol. 18, no. 2, pp. 151-160, 1996.

[6] F. Mai, Y. S. Hung, and G. Chesi, "Projective reconstruction of ellipses from multiple images," Pattern Recogn., vol. 43 , no. 3, pp. 545-556, 2010.

[7] J. Y. Kaminski and A. Shashua, "On calibration and reconstruction from planar curves," in ECCV '00: Proceedings of the 6th European Conference on Computer Vision-Part I. London, UK: Springer-Verlag, 2000, pp. 678-694.

[8] - "Multiple view geometry of general algebraic curves," International Journal of Computer Vision, vol. 56, no. 3, pp. 195-219, 2004.

[9] R. Berthilsson and K. Åström, "Extension of affine shape," Journal of Mathematical Imaging and Vision, vol. 11, no. 2, pp. 119-136, 1999.

[10] R. Berthilsson, K. Åström, and A. Heyden, "Reconstruction of general curves, using factorization and bundle adjustment," International Journal of Computer Vision, vol. 41, no. 3, pp. 171-182, 2001.

[11] $\mathrm{H} . \mathrm{Wu}$ and $\mathrm{Y}$. Yu, "Photogrammetric reconstruction of freeform objects with curvilinear structures," The Visual Computer, vol. 21, no. 4, pp. 203-216, 2005.

[12] J. dong Zheng, L. yan Zhang, X. yu Du, and Z. an Ding, "3d curve structure reconstruction from a sparse set of unordered images," Computers in Industry, vol. 60, no. 2, pp. 126-134, 2009.

[13] R. I. Hartley and A. Zisserman, Multiple View Geometry in Computer Vision. Cambridge University Press, ISBN: 0521623049, 2000.

[14] Y. S. Hung and W. K. Tang, "Projective reconstruction from multiple views with minimization of $2 \mathrm{~d}$ reprojection error," Int. J. Comput. Vision, vol. 66, no. 3, pp. 305-317, 2006.

[15] F. Mai, C. Chang, and Y. Hung, "Affine-invariant shape matching and recognition under partial occlusion," in ICIP, 2010 .

[16] F. Mokhtarian and S. Abbasi, "Shape similarity retrieval under affine transforms," Pattern Recognition, vol. 35, no. 1, pp. 31$41,2002$.

[17] M. S. Waterman and T. F. Smith, "Identification of common molecular subsequences." J. Mol. Biol., vol. 147, pp. 195197, 1981.

[18] C. Li, C. Kao, J. Gore, and Z. Ding, "Implicit active contours driven by local binary fitting energy," in Proceedings of IEEE Conference on Computer Vision and Pattern Recognition $(C V P R)$. Washington, DC, USA: IEEE Computer Society, 2007, pp. 1-7. 